\title{
DI-3-n-butylphthalide improves neurological function of a cardiac arrest patient with hypoxic-ischemic encephalopathy: a case report
}

\author{
Fei Chen ${ }^{1,2,3,4} \cdot$ Ming-Hao Yuan ${ }^{1,2,3,4} \cdot$ Yun-wen $\mathrm{Qi}^{1,2,3,4} \cdot$ Yong-ling Zheng ${ }^{2} \cdot$ Zhi-you Cai $^{1,2,3,4,5}$
}

Received: 20 April 2021 / Accepted: 18 July 2021 / Published online: 31 July 2021

(c) Fondazione Società Italiana di Neurologia 2021

\section{Dear Editor,}

With the spread of cardiopulmonary resuscitation (CPR) education, $20-50 \%$ of patients with cardiac arrest (CA) can obtain spontaneous breathing through CPR. However, merely 2 to $14 \%$ of patients can be discharged successfully, due to secondary acute ischemic hypoxicischemic encephalopathy (HIE). Based on previous researches, the brain is extremely sensitive to ischemia and hypoxia. Even if the patients' spontaneous circulation is restored after CPR, most patients suffered from irreversible craniocerebral injuries such as different degrees of learning/memory deficits, decreased response, and intelligence decline. Hypoxic-ischemic encephalopathy and its complication mainly contribute to poor prognosis of patients. We report a case of hypoxic-ischemic encephalopathy after CPR treated with Dl-3-n-butylphthalide (NBP). This case highlights the potential therapeutic benefit of the NBP treatment to improve neurological function and activities of daily living (ADL).

\section{Zhi-you Cai}

caizhiyou@ucas.ac.cn

1 Chongqing Medical University, 400042 Chongqing, China

2 Department of Neurology, Chongqing General Hospital, University of Chinese Academy of Sciences, No. 118, Xingguang Avenue, Liangjiang New Area, Chongqing 401147, China

3 Chongqing School, University of Chinese Academy of Sciences, Chongqing 400714, China

4 Chongqing Key Laboratory of Neurodegenerative Diseases, Yuzhong District, Chongqing No. 312, Zhongshan First Road, Chongqing 400013, China

5 Department of Neurology, Chongqing General Hospital, University of Chinese Academy of Sciences, Yuzhong District, No. 104, Pipashan Main Street, Chongqing 400014, China
A 28-year-old man who is an electrician collapsed in supine position while repairing the telephone line owing to a $220-\mathrm{V}$ electric shock. Immediately, he lost his consciousness with no pulse or breathing. He received $30 \mathrm{~min}$ of preclinical non-professional CPR until being hospitalized on an acute neurological intensive care unit. Before being presented to our hospital, this patient has hospitalized for 14 days in two different medical facilities. Only the support conventional symptomatic treatment of neurology was given. He was under assisted ventilation via a tracheal cannula and in persistent comatose. His clinical courses including ventilator-associated pneumonia with $\mathrm{Pseu}$ domonas aeruginosa, urinary catheter-related infection, and intensive care unit-acquired weakness are extremely complicated. On admission to our neurological intensive care unit, neurological examination showed his Glasgow Coma Scale was E1TD1, and cerebral performance category was 4 grading. A noncontrast head CT was performed, which implied sporadic cerebral cortex hyperintensity suggesting HIE (Fig. 1). The electroencephalography report showed a low-voltage EEG, which indicated the poor prognosis for recovery after HIE. The representative EEGs are shown in Fig. 2. The patient had serious post-anoxic myoclonus and secondary parkinsonism (e.g., dystonia). These features implied a poor prognosis of consciousness $[1,2]$.

On the day of admission to our acute intensive care, he was under medication treatment with levetiracetam (1000 mg per day), valproate (1200 mg per day), piribedil (150 mg per day), levodopa (600 mg per day), and benserazide (100 mg per day). A course of hyperbaric oxygen therapy also was initiated. Seventeen days after his collapse, we began treatment with NBP medications $(400 \mathrm{mg} / \mathrm{day})$ through canalis ventriculi. On day 
Fig. 1 A non-contrast head CT, 18 days after post-electric injury; $\mathbf{a}, \mathbf{b}$, and $\mathbf{c}$ show the widespread and bilateral hemispheric distribution of slightly high-density shadows with the most obvious in occipital lobe
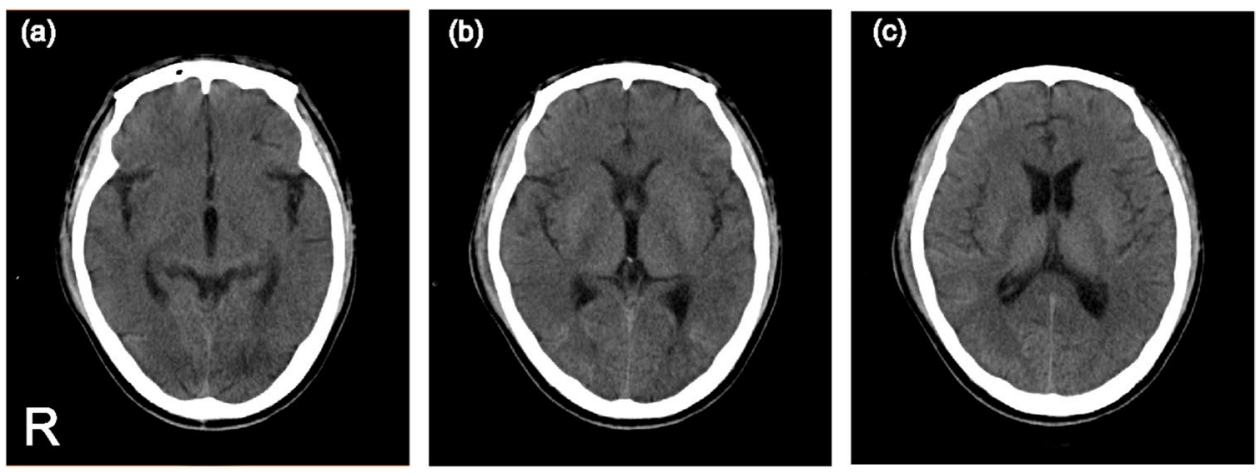

Fig. 2 The EEG of the patient upon first admission. a Electroencephalogram (EEG) performed showed the EEG leads were based on the low-voltage waves activity with a frequency of 4.0-6.0 Hz with no evidence of alpha wave
48 post-electric injury coma, the patient woke up and recognized his wife and could smile. He can also perform simple actions such as a nod, shrug, and finger extension. However, his right hand had frequent tremors (frequency of $6 \mathrm{~Hz}$ ). After extubation, he could answer simple questions like his name and where he was. Considering the remarkable benefits of NBP, intravenous transfusion administration of $100 \mathrm{ml}$ NBP once a day was initiated on December 10. Twelve weeks after admission to our hospital, he was discharged home with a full Glasgow Coma Scale and a cerebral performance category of 2 and the frequency of his right-hand tremor had decreased visibly. After NBP medications, progressive improvements were shown in his neurological recovery. The patient in follow-up EEG showed improvement (Fig. 3). He could perform some simple behaviors such as dressing, stair activity and going to toilet, and eating with minor assistance. The detailed treatment and clinical course are shown in Fig. 4. After 
Fig. 3 The follow-up EEG of the patient on Nov. 17. b Electroencephalogram (EEG) performed 8 weeks after NBP treatment showed that the EEG leads were predominately based on low-medium wave activity at a frequency of $4.0-7.0 \mathrm{~Hz}$, which was distributed diffusely signals in moderately abnormal EEG. While there was still no evidence to monitor the alpha waves, the situation was improved than previous EEG

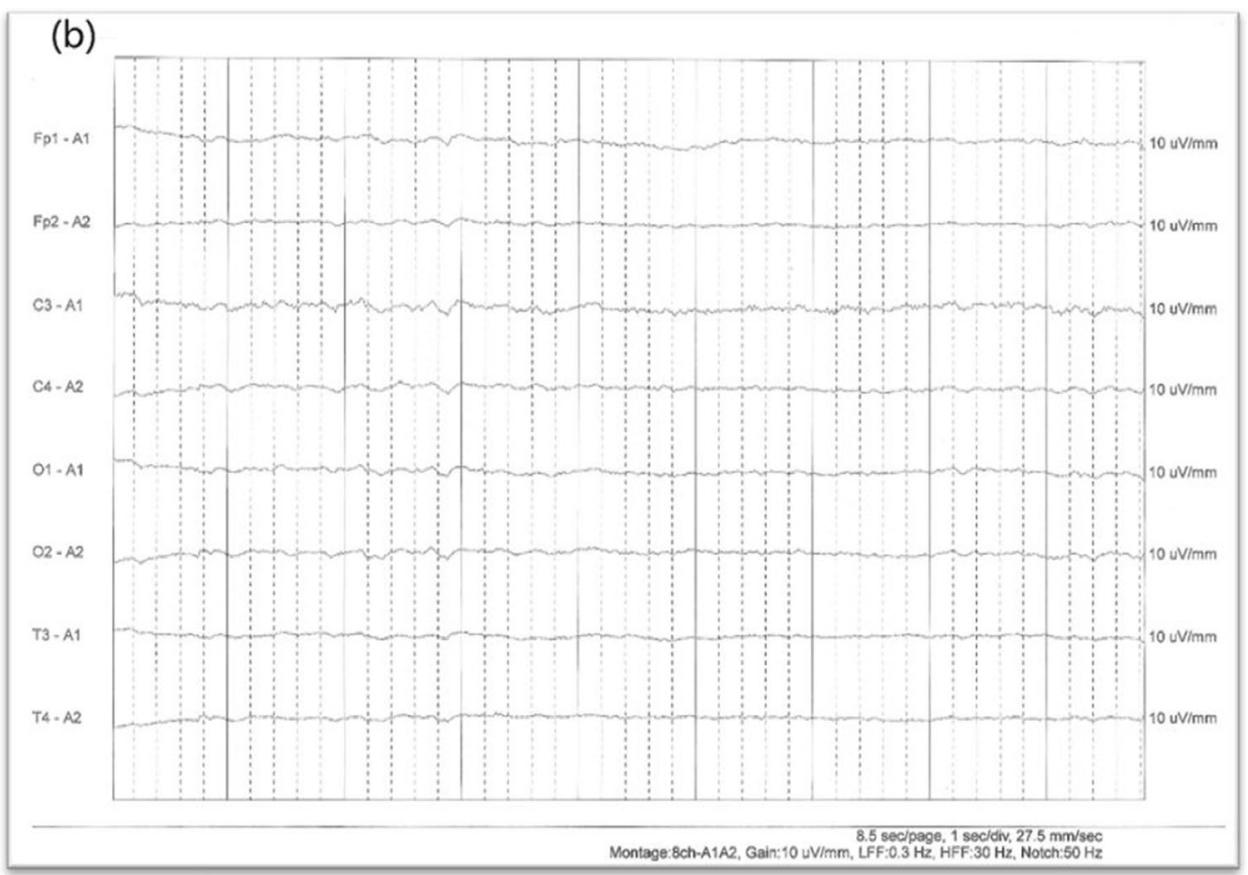

12 weeks of follow-up of this case, his right-hand tremor markedly improved and the level of ADL remained.

The physiopathologic mechanism of HIE exerts cognitive impairment is unclear. Recent evidence suggests that the consecutive dysfunction of neuronal membrane pumping and membrane depolarization lead to influx of calcium, lactate acidosis, glutamate release, occurrence of free fatty acids, and excitatory amino acids which cause damages to neurons, resulting in cognitive, sensory, motor dysfunction, and emotional function disorders [3,4]. Overall, the growing evidence seems to suggest that cerebrovascular accidents and HIE may have common pathophysiological mechanisms.

NBP, a small molecular substance, extracted from the celery seeds reduce cerebral edema and improve cerebral blood flow. From previous studies, it is known that NBP acts on multiple mechanisms, such as oxidative stress and mitochondrial dysfunction apoptosis and inflammation, and is extensively used by Chinese caregivers to help improve the outcome of patients with stroke. In addition, a number of recent studies showed that the benefits of NBP may be partly more important than the management of stroke.

Several studies have shown that treatment for an ischemic cerebrovascular disease with NBP can promote the formation of new blood vessels around the lesion site and the regeneration of neuro vessels in the periinfarct area. Additionally, NBP can increase the number of RECA-1 positive vessels and enhance the expression of tight junction protein occludin by HIF- $1 \alpha$-VEGF and Notch-Dll4 upregulation. Previous studies have shown that NBP can improve cognitive impairment through antioxidant stress and anti-inflammatory, protecting the integrity of blood-brain barrier, reducing autophagy, and increasing synaptic plasticity [5]. In addition, a recent asphyxia-induced cardiac arrest followed by a cardiopulmonary resuscitation mice model has shown that NBP can reduce the ultrastructural lesion in mitochondria [6].

Treating with the HIE needs comprehensive therapy which has been confirmed by former research. Although diagnosis criteria for HIE have been developed, no guidelines for long-term therapeutic strategies are established. According to the outcome of this patient, NBP could serve as an alternative method integrating with conventional therapy (e.g., HBOT), providing us a reason for further research. Meanwhile, NBP may be inferred as a disease-modifying therapy for Parkinson's disease or other neurodegenerative diseases. While we can not discount that the HBOT helps recovery of neural function, our clinical experience has seen that this patient may prolong the time-to-conscious regaining and 


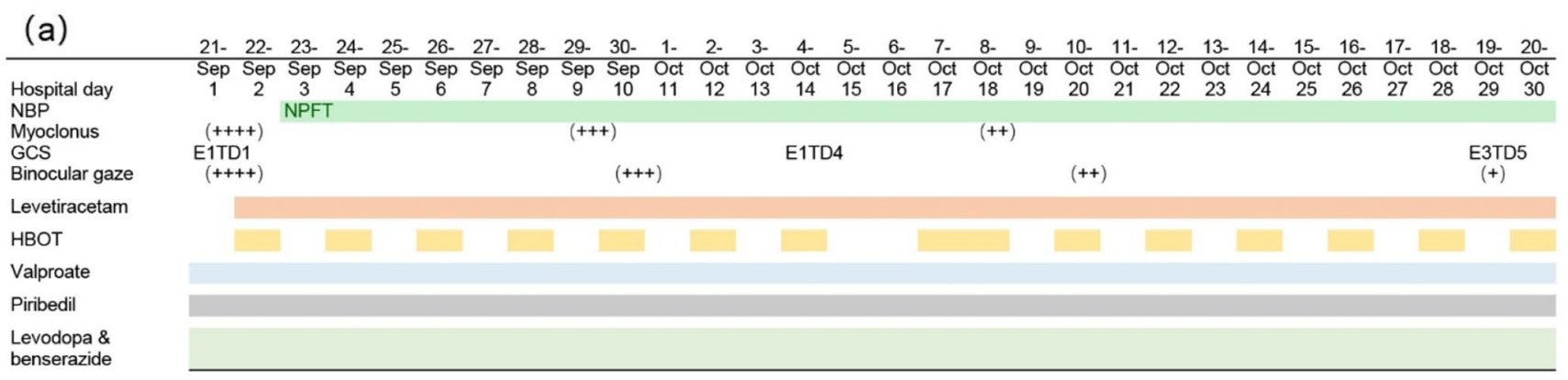

(b)

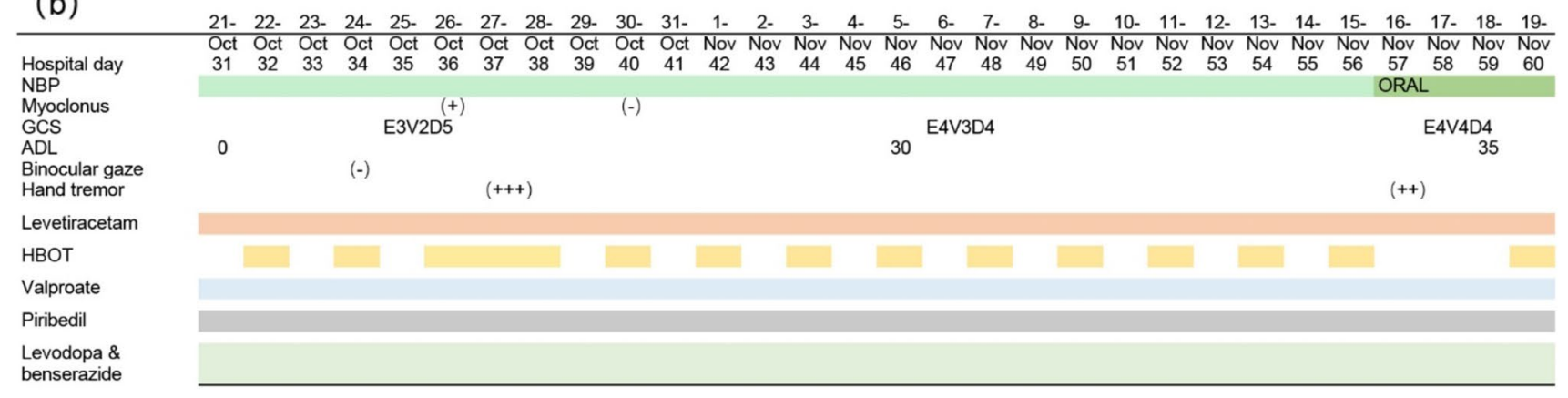

(c)

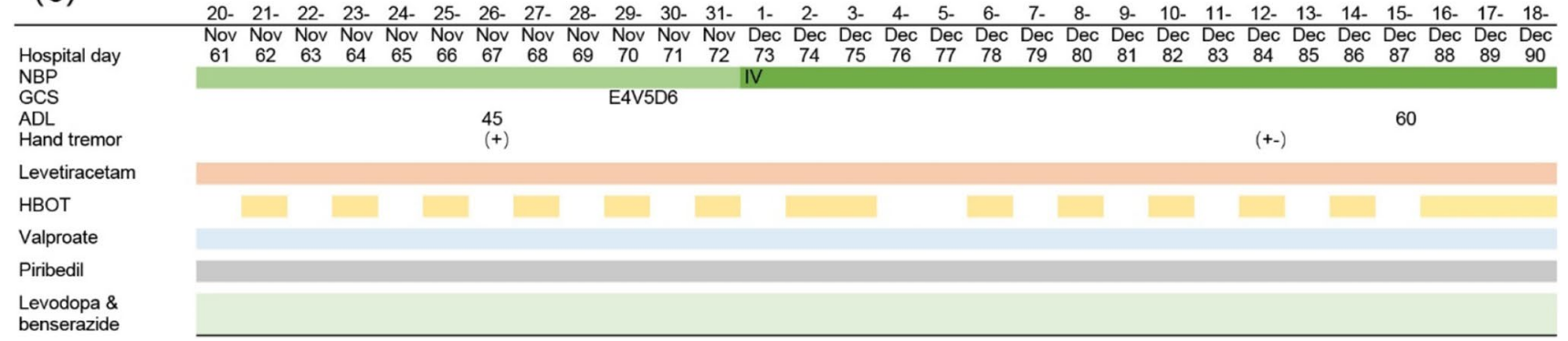

Fig. $4 \mathbf{a}, \mathbf{b}$, and $\mathbf{c}$ show the clinical course and treatment of the case HIE. HBOT $=$ hyperbaric oxygen treatment, $+\sim-=$ the frequency and severity of symptom, NPFT = nasally placed feeding tube, IV = intravenous

the restoration of cognitive ability and impaired nerve function without NBP, which has successfully improved his ADL.

\section{Declarations}

Ethical approval The Institutional Review Board at Chongqing General Hospital approved this case report.

Informed consent Written informed consent for publication of this case report was obtained from the wife of the patient.

Conflict of interest The authors declare no competing interests.

\section{References}

1. Krumholz A, Stern BJ, Weiss HD (1988) Outcome from coma after cardiopulmonary resuscitation: relation to seizures and myoclonus. Neurology 38(3):401-405. https://doi.org/10.1212/wnl.38.3.401

2. Szczepanska A, Dziadkowiak E, Bladowska J, Kipinski L, Budrewicz S, Koszewicz M (2019) The usefulness of quantitative EEG and advanced MR techniques in the monitoring and long-term prognosis of Lance-Adams syndrome. Front Neurol 10:214. https://doi.org/10.3389/fneur.2019.00214

3. Ahn SY, Chang YS, Sung DK, Sung SI, Park WS (2018) Hypothermia broadens the therapeutic time window of mesenchymal stem cell transplantation for severe neonatal hypoxic ischemic encephalopathy. Sci Rep 8(1):7665. https://doi.org/10.1038/ s41598-018-25902-x

4. Li B, Dasgupta C, Huang L, Meng X, Zhang L (2020) MiRNA-210 induces microglial activation and regulates microglia-mediated 
neuroinflammation in neonatal hypoxic-ischemic encephalopathy. Cell Mol Immunol 17(9):976-991. https://doi.org/10.1038/ s41423-019-0257-6

5. Huang L, Lan J, Tang J, Kang Y, Feng X, Wu L, Peng Y (2021) L-3-n-Butylphthalide improves synaptic and dendritic spine plasticity and ameliorates neurite pathology in Alzheimer's disease mouse model and cultured hippocampal neurons. Mol Neurobiol 58(3):1260-1274. https://doi.org/10.1007/s12035-020-02183-y

6. Yang S, Yu C, Yang Z, Cui H, Wu Y, Liang Z, Liu Y, Shi X, Shao F, Zhao S, Tang Z (2021) DL-3-n-butylphthalide-induced neuroprotection in rat models of asphyxia-induced cardiac arrest followed by cardiopulmonary resuscitation. J Cell Physiol. https:// doi.org/10.1002/jcp.30442

Publisher's note Springer Nature remains neutral with regard to jurisdictional claims in published maps and institutional affiliations. 